\title{
Masers as probes of massive star formation in the nuclear disk
}

\author{
F. Yusef-Zadeh ${ }^{1}$, R. G. Arendt ${ }^{2}$, C. O. Heinke ${ }^{1}$, J. L. Hinz ${ }^{3}$, J. W.

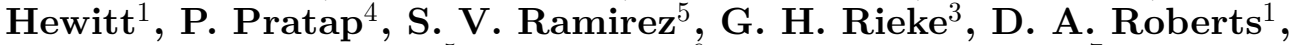 \\ S. R. Stolovy ${ }^{5}$, M. Wardle ${ }^{6}$ and B. A. Whitney ${ }^{7}$ \\ ${ }^{1}$ Department of Physics and Astronomy, Northwestern University, Evanston, IL 60208 USA \\ email: zadeh@northwestern.edu,j-hewitt@northwestern.edu, cheinke@northwestern.edu, \\ doug-roberts@northwestern.edu \\ ${ }^{2}$ CRESST/UMBC/GSFC, Code 665, Greenbelt, MD 20771 USA \\ email: arendt@milkyway.gsfc.nasa.gov \\ ${ }^{3}$ Steward Observatory, University of Arizona, 933 N. Cherry Ave., Tucson, AZ 85721 USA \\ email: grieke@as.arizona.edu, jhinz@as.arizona.edu \\ ${ }^{4}$ MIT Haystack Observatory, Westford, MA 01886 USA \\ email: ppratap@haystack.mit.edu \\ ${ }^{5}$ IPAC, Cal Tech, Pasadena, CA 91125 USA \\ email: solange@ipac.caltech.edu, stolovy@ipac.caltech.edu \\ ${ }^{6}$ Department of Physics, Macquarie University, Sydney NSW 2109, Australia \\ email:wardle@ics.mq.edu.au \\ ${ }^{7}$ Space Science Institute, 4750 Walnut Street, Suite 205, Boulder, CO 80301 USA \\ email: bwhitney@spacescience.org
}

\begin{abstract}
OH}(1720 \mathrm{MHz})$ and methanol masers are now recognized to be excellent probes of the interactions of supernova remnants with molecular clouds and tracers of massive star formation, respectively. To better understand the nature of star formation activity in the central region of the Galaxy, we have used these two classes of masers combined with the IRAC and MIPS data to study prominent sites of ongoing star formation in the nuclear disk. The nuclear disk is characterized by massive GMCs with elevated gas temperatures, compared to their dust temperatures. We note an association between methanol masers and a class of mid-infrared "green sources". These highly embedded YSOs show enhanced $4.5 \mu \mathrm{m}$ emission due to excited molecular lines.

The distribution of methanol masers and supernova remnants suggest a low efficiency of star formation (with the exception of Sgr B2), which we believe is due to an enhanced flux of cosmic ray electrons impacting molecular clouds in the nuclear disk. We also highlight the importance of cosmic rays in their ability to heat molecular clouds, and thus increase the gas temperature.
\end{abstract}

Keywords. Masers, supernova remnants, cosmic rays.

\section{Introduction}

Understanding the processes occurring in the nuclear disk of our own Galaxy is interesting not only for insight into our own Milky Way Galaxy, but also because it is the closest galactic nucleus. This important region of the Galaxy hosts several sources of energetic activity, and is the site of massive molecular clouds with pockets of past and present massive star formation. However, the study of the nuclear disk of our galaxy has led to a number of paradoxes, puzzles and discrepancies. One of the best paradoxical examples is related to the nature of the compact radio source $\mathrm{Sgr} \mathrm{A}^{*}$ at the dynamical center of the Galaxy. Although there is compelling evidence that Sgr A* is a massive black hole (Schödel et al. 2003; Ghez et al. 2003), its X-ray luminosity is orders of magnitude lower than expected. Another paradox is related to the expectation that the formation 
of stars near Sgr A* should be forbidden due to the extreme tidal forces exerted by Sgr $\mathrm{A}^{*}$; however, many young stars are distributed in a disk orbiting Sgr A* (Paumard et al. 2006). Another outstanding puzzle is the origin of nonthermal filaments in the Galactic nucleus, which has not been understood since their discovery (see Yusef-Zadeh, Hewitt \& Cotton 2004; Nord et al. 2004).

On a larger scale, the nature of on-going star formation in the nuclear region remains unclear. Although there is a high concentration of dense molecular clouds distributed in this region, the star formation rate has shown extreme values. On the one hand, the massive star forming region Sgr B2 points to the closest example of starburst activity in our Galaxy, whereas the quiescent giant molecular cloud (GMC) G0.25+0.01 (Lis \& Carlstrom 1994; Lis \& Menten 1998) appears to contain highly inefficient star formation. This region contains some of the most spectacular molecular clouds with elevated gas temperatures but no obvious source of heating of the clouds. In the last two decades, studies of this region have indicated that the molecular gas is warm, ranging between 75 to $200 \mathrm{~K}$ (e.g., Hüttemeister et al. 1993). However, studies based on far-IR observations have revealed dust temperature to be $<\sim 30 \mathrm{~K}$ (Odenwald \& Fazio 1984; Cox \& Laureijs 1989; Pierce-Price et al. 2000). This discrepancy between the gas and dust temperatures in the central region of the Galaxy is puzzling.

Using SNR masers and methanol masers, we place constraints on the low efficiency of star formation in the Galactic nucleus. We then argue that the low efficiency of star formation and the elevated gas temperature with respect to dust temperature can be explained by the enhanced energy density of cosmic rays interacting with molecular clouds in the nuclear disk. The interstellar medium of the nuclear disk is distributed in the region with an extent $-1^{0}<l<2^{0}$ and $-0.5^{0}<b<0.5^{0}$ and is characterized by a strong concentration of molecular gas in the so-called "Central Molecular Zone" with a radius of $\sim 200 \mathrm{pc}$ (Morris \& Serabyn 1996). Physical conditions in this region are extreme, characterized by high velocity dispersion $(\sim 20 \mathrm{~km} / \mathrm{s})$, high density $\left(\sim 10^{4}\right.$ $\left.\mathrm{cm}^{-3}\right)$ and high column density $\left(\sim 10^{23} \mathrm{~cm}^{-2}\right)$.

\section{Supernova remnant masers}

The high number density as well as the high column density of warm gas in the nuclear disk are similar to the restricted physical conditions required to produce $\mathrm{OH}(1720$ $\mathrm{MHz}$ ) masers (Lockett, Gauthier \& Elitzur 1999). At the site of C-type shocked gas, $\mathrm{OH}$ abundance is expected to be enhanced sufficiently to detect $\mathrm{OH}(1720 \mathrm{MHz})$ maser emission throughout the nuclear disk (Wardle 1999). With this in mind, we carried out an unbiased, snapshot survey of the Galactic nucleus to search for SNR masers. When this survey is combined with other targeted surveys, a total of six SNRs are detected toward roughly the inner $8^{0} \times 1^{0}$ (Yusef-Zadeh et al. 1995, 1996, Frail et al. 1996). However, only four SNR masers G0.0+0.0 (Sgr A East), Sgr D (G1.13-0.1), G1.4-0.1 \& G359.1-0.5 are detected toward the nuclear disk. The only known SNRs with no maser counterparts in the nuclear disk are G0.9+0.1 and G0.33+0.01. Here we discuss SNR masers associated with the SNRs Sgr A East and G359.1-0.5.

\subsection{SNR G0.0+0.0}

The most well known interacting SNR in the nuclear disk is Sgr A East (SNR G0.0+0.0) projected toward Sgr A*. The kinematics of $\mathrm{OH}(1720 \mathrm{MHz})$ masers indicate two populations. The first kinematic population has velocities ranging between 30 and $60 \mathrm{~km} \mathrm{~s}^{-1}$ distributed around the shell of Sgr A East. Recent sensitive observations of $\mathrm{OH}$ masers toward Sgr A East have discovered a number of additional maser spots (see the references 

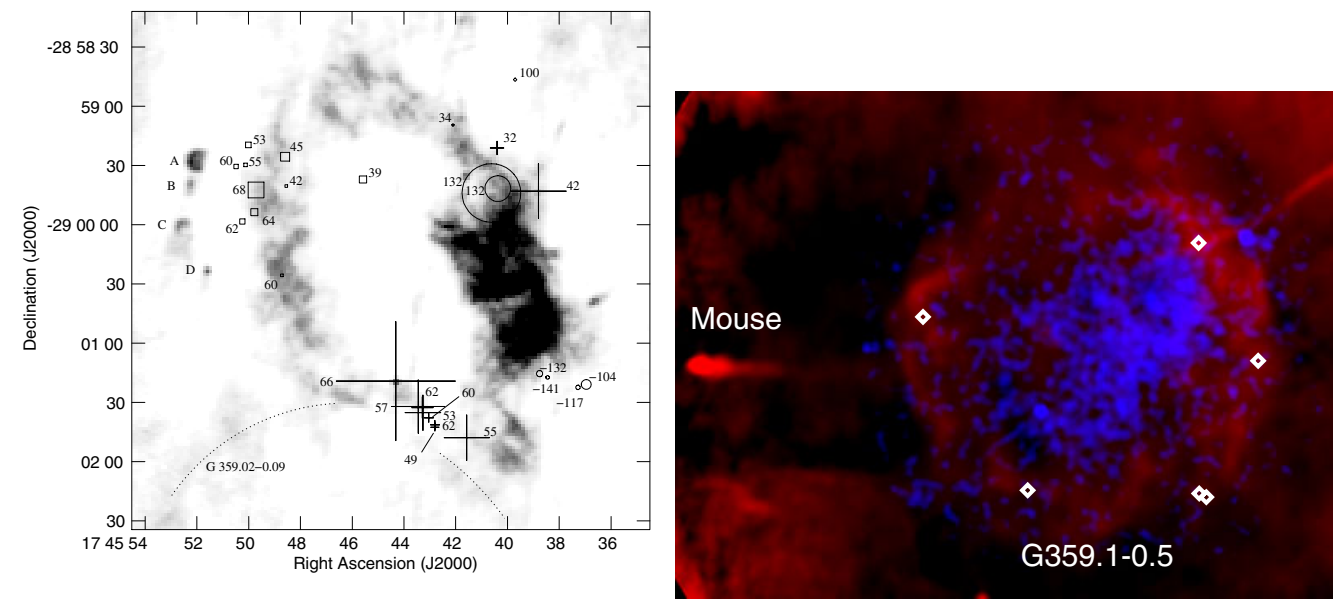

Figure 1. (a) A grayscale image of the continuum emission from Sgr A East and West at $20 \mathrm{~cm}$. The distribution of $\mathrm{OH}(1720 \mathrm{MHz})$ maser spots with their $\mathrm{V}_{L S R}$ velocities are superimposed on the nonthermal Sgr A East shell and the thermal spiral-like structure of Sgr A West (taken from Figure 1 of Pihlström and Sjouwerman 2006). (b) A 20cm continuum image of G359.1-0.5 (red color) shows the shell-like structure of this remnant. The interior of the shell is represented by the distributions of X-ray (blue color). The diamond points represent the positions of maser spots. The elongated feature to the east of the figure corresponds to radio emission from the pulsar wind nebula known as the Mouse. A colour rendition of this figure can be found in the on-line version.

within Pihlstrom and Sjouwerman 1996; Karlsson et al. 2003). These measurements established that Sgr A East is interacting with molecular gas associated with the $50 \mathrm{~km} \mathrm{~s}^{-1}$ GMC. A number of weak $\mathrm{OH}(1720 \mathrm{MHz})$ masers to the northeast of Sgr A East coincide with a large number of shocked molecular clumps, as probed by CS (1-0) line observation with NMA (Tsuboi et al. 2006), The broad line emission from molecular gas provides additional support for shocked $50 \mathrm{~km} \mathrm{~s}^{-1}$ clouds as a result of the expansion of the SN shock. The line profiles of the CS line emission are asymmetrically blue shifted which implies that the far side of the the $50 \mathrm{~km} \mathrm{~s}^{-1}$ GMC is being shocked by the expansion of the Sgr A East shell located behind the $50 \mathrm{~km} \mathrm{~s}^{-1}$ cloud (Tsuboi et al. 2006).

The second kinematic component shows highly blue- and red-shifted velocities at $\pm 132 \mathrm{~km} \mathrm{~s}^{-1}$ (Yusef-Zadeh et al. 2002; Pihlström and Sjouwerman 2006). These high velocity masers are distributed symmetrically at the tangent points of the lobes of the circum-nuclear ring orbiting Sgr A* $\mathrm{OH}(1720 \mathrm{MHz})$ masers are excellent indicators of the systemic motion of the molecular gas that is being shocked. The low velocity dispersion noted between the highly blue- and red-shifted masers places a constraint on the average motion of the enclosed material within the ring of $\mathrm{V}_{L S R} \sim 0 \mathrm{~km} \mathrm{~s}^{-1}$. The origin of the masers associated with the circum-nuclear ring remains less clear than those of Sgr A East. On the one hand, it is possible that the expansion of Sgr A East runs into the circum-nuclear ring and produces the observed $\mathrm{OH}(1720 \mathrm{MHz})$ masers. This is consistent with the fact that Sgr A East lies behind Sgr A* (Pedlar et al. 1989). In this picture, Sgr A East lies behind both the $50 \mathrm{~km} \mathrm{~s}^{-1}$ molecular cloud and the circumnuclear molecular ring and is responsible for production of $\mathrm{OH}(1720 \mathrm{MHz})$ masers in both clouds. On the other hand, these high velocity maser spots may originate within the ring by alignment of molecular clumps, resulting in amplification along the line of sight (Pihlström \& Sjouwerman 2006). Future study of these masers should clarify their relationship to the circum-nuclear molecular ring which is known to be orbiting Sgr A*. 


\subsection{SNR G359.1-0.5}

G359.1-0.5 shows several masers surrounding the remnant at a velocity of $-5 \mathrm{~km} \mathrm{~s}^{-1}$ and a low velocity dispersion. Such low velocity maser features are unusual if the remnant is interacting with molecular gas in the nuclear disk. This low velocity is in contrast to the kinematics of a CO shell suggested to surround this remnant, which shows velocities ranging between -60 and $-150 \mathrm{~km} \mathrm{~s}^{-1}$ (Uchida et al. 1992). Several more recent studies such as the detections of a bar of $\mathrm{H}_{2}$ emission at low velocities and the linear polarization from the brightest maser spot as well as the relatively low rotation measure of the remnant itself indicate that G359.1-0.5 is unlikely be located in the Galactic nucleus. More recently, an XMM observation of thermal X-ray gas within the remnant supports a low column density to the remnant, consistent with it being a foreground source (Heinke et al. 2007, in preparation), as described below. These observations indicate clearly that $\mathrm{OH}(1720$ $\mathrm{MHz}$ ) maser emission is associated with a foreground remnant. This implies that $\mathrm{OH}(1720$ $\mathrm{MHz}$ ) masers are powerful indicators of the systemic motion of the interacting remnant especially in the confused region of the nuclear disk.

The overall fit to the X-ray emission from G359.1-0.5 places a strong constraint on the low-energy absorption, $\mathrm{N}_{H}=2.6 \pm 0.2 \times 10^{22} \mathrm{~cm}^{-2}$ (Heinke et al. 2007, in preparation). The pulsar wind nebula known as the Mouse lies only 22 arcminutes from the center of the remnant, and pulsar dispersion measurements infer a distance to the Mouse between 3 and $5.5 \mathrm{kpc}$ (requiring the Mouse to be within the molecular ring at 3-5 kpc distance, but not beyond the spiral arm $3 \mathrm{kpc}$ from the Galactic center), giving a nominal value of $5 \mathrm{kpc}$. The $\mathrm{N}_{H}$ to the Mouse is also estimated from X-ray measurements to be $2.60 \pm 0.09 \times 10^{22}$ $\mathrm{cm}^{-2}$ (Mori et al. 2004; Gaensler et al. 2004). Our measured $\mathrm{N}_{H}$ value is consistent with the Mouse's $\mathrm{N}_{H}$, suggesting that G359.1-0.5 is located at a similar distance and is likely to be the origin of the Mouse.

\subsection{Star formation rate from known SNRs}

If we remove G359.1-0.5 from the list of remnants interacting with molecular gas in the nuclear disk, then $3 / 5$ or $\sim 60 \%$ of all known remnants in this region are interacting with molecular gas in the nuclear disk. This is in contrast to the fraction of interacting remnants in the disk of the Galaxy, 10\%. This implies a uniform distribution of molecular gas throughout the nuclear disk.

Given the number of known SNRs and assuming a residence time of $2.5 \times 10^{4} \mathrm{yrs}$, the SN rate is estimated to be one per 5000 years. This implies that the rate of formation of massive stars is $10^{-2} \mathrm{M}_{\odot} \mathrm{yr}^{-1}$ (Condon 1992). This is consistent with the far-IR measurements inferring a paucity of massive stars in the nuclear disk (Odenwald and Fazio 2004). This estimate is, however, quite uncertain since the residence time of a remnant expanding into a dense medium is unknown. If the residence time is shorter than the above estimate, the star formation rate estimate increases, accordingly.

\section{Methanol masers}

It is now well established that methanol masers are signposts of ongoing massive star formation throughout the Galaxy. A survey of the inner 2 degrees of the Galactic center showed a total of 23 class II methanol masers at $6.7 \mathrm{GHz}$ (Caswell 1996). This class of masers is recognized to be radiatively pumped, unlike class I methanol masers, known to be collisionally pumped (e.g., Menten 1991). There is a strong correlation between the 6.7 GHz methanol masers and infrared dark clouds as revealed in the $8 \mu \mathrm{m}$ Spitzer observations of the Galactic center (Stolovy et al. 2006). The infrared dark clouds are likely progenitors of high mass stars, and the masers indicate locations where star formation 

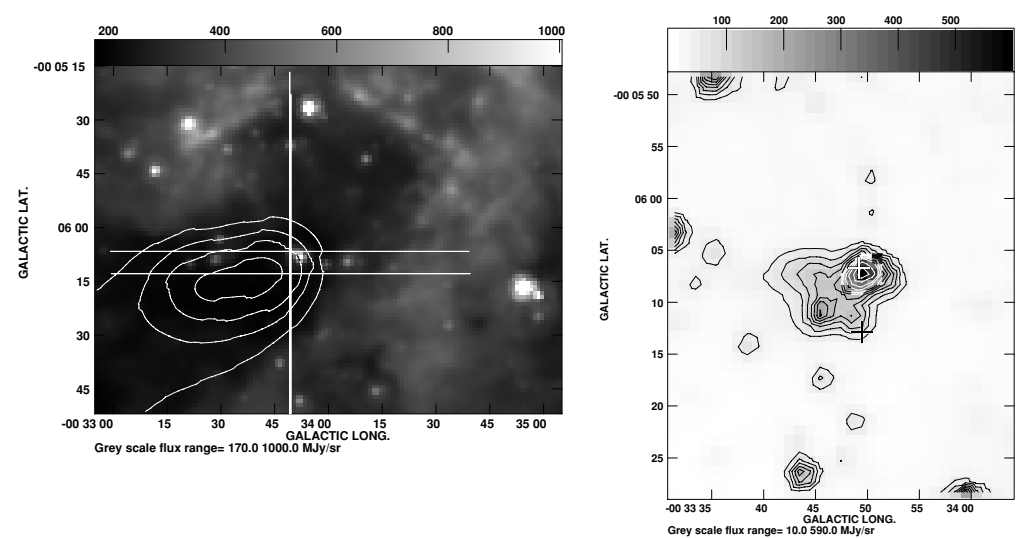

Figure 2. (a) Contours of $850 \mu \mathrm{m}$ emission (Pierce-Price et al. 2000) are superimposed on the $8 \mu \mathrm{m}$ image of Sgr C. (b) Contours of $4.5 \mu \mathrm{m}$ emission based on Channel 2 of IRAC data. The crosses in (a) and (b) represent methanol maser distributions associated with Sgr C.

is currently taking place. Closer examination of the kinematics of the $6.7 \mathrm{GHz}$ methanol masers indicates that 11 maser spots arise from Sgr B2, two from Sgr C, and 3-5 from the quiescent dust ridge G0.25-0.01. Given that there is a large reservoir of dense molecular gas, up to $10^{8} \mathrm{M}_{\odot}$, distributed in the nuclear disk, there appears to be a relative paucity of methanol masers (above $1 \mathrm{Jy}$ ) in the nuclear disk (Caswell 1996). With the exception of Sgr B2, the rarity of bright $6.7 \mathrm{GHz}$ masers suggests low star formation activity in the nuclear disk. The inner 30 pcs of the Galactic center show no evidence of any class II methanol masers. Remarkably, this inner region is known to have a large concentration of dense giant molecular clouds associated with the 20, 40 and $-30 \mathrm{~km} \mathrm{~s}^{-1}$ molecular clouds (e.g., Güsten et al. 1981). As described below, this region is also known to be coincident with strong nonthermal emission from Sgr A East, its halo and the radio continuum arc near $l \sim 0.2^{0}$.

\section{1. "Green" sources}

To determine if any of the methanol masers have a stellar counterpart in the mid-IR, we examined the color of stars in the nuclear disk. One of the most powerful methods of detecting molecular line emission in mid-IR wavelengths using IRAC images is to look for excess emission at $4.5 \mu \mathrm{m}$ ("green" in 3-color images combining 3.6, 4.5 and 8 $\mu \mathrm{m}$ emission). Using this color selection criterion, a recent IRAC survey of the Galactic plane has identified a number of interacting supernova remnants showing excess emission at $4.5 \mu \mathrm{m}$, indicating shocked, excited $\mathrm{CO}$ and $\mathrm{H}_{2}$ molecular line emission (Reach et al. 2006). Also, the "green nebulosity" has been detected in DR21, a massive star formation site, in which the excess $4.5 \mu \mathrm{m}$ color is considered to be due shocked molecular outflow (Marston et al. 2004; Smith et al. 2006).

Using mid-IR data, we have examined the color of stars and dust clouds in star forming sites throughout the nuclear disk. The infrared images of the nuclear disk are generated from the IRAC Galactic center survey mosaics (Stolovy et al. 2006). A quantified indication of excess $4.5 \mu \mathrm{m}$ emission is obtained by constructing the ratio $I(4.5) /[1.2 * I(3.6) * I(5.8)]^{(0.5)}$. This essentially is the ratio of the actual $4.5 \mu \mathrm{m}$ intensity to that determined by a power-law interpolation of the 3.6 and $5.8 \mu \mathrm{m}$ intensities. It was found empirically that a slight $(\sim 3 \%)$ modification to the interpolation coefficients helps emphasize sources with unusually strong $4.5 \mu \mathrm{m}$ emission. In a map of this ratio across the entire Galactic center survey, most point sources exhibit a uniform ratio that is not 
very different from the background. In regions of high extinction (e.g. IR dark clouds, and Sgr B2), this ratio rises because the reddening reduces the $3.6 \mu \mathrm{m}$ intensity. However, there were of order 100 sources, located in regions of both high and low extinction, which exhibit ratios distinctly higher than those of other sources in their vicinity (within several arc minutes). These include 33 sources that were visually selected as "green" sources in 3 -color images. Yet the ratio also clearly identifies a number of interesting sources that do not stand out in the 3 -color images, such as 3 planetary nebulae. There are thirty green sources suggesting YSOs embedded within infrared dark clouds. A correlation of methanol masers in the nuclear disk with the green sources has led to the surprising result that about $1 / 3$ of green sources have maser counterparts, $1 / 3$ have no maser counterparts, and the rest have not yet been observed. Given the limited sensitivity of 1 Jy in the maser survey by Caswell (1996), it is possible that many of the green sources have weak maser counterparts or that they signify early sites of massive star forming regions.

Although the $\sim 2^{\prime \prime}$ spatial resolution of IRAC images being different than the $2^{\prime \prime} \times 4^{\prime \prime}$ resolution of methanol maser observations (Caswell 1996), we have mapped the data with $1^{\prime \prime}$ pixels. The green sources are generally seen to be extended on a scale $\sim 10^{\prime \prime}$ and the maser sources fall within the extent of the $4.5 \mu \mathrm{m}$ emission. An example in which the relationship between a green source and a methanol maser can be seen is in the Sgr C complex region. This complex is known to consist of an evolved, extended HII region and a prominent nonthermal radio filament running perpendicular to the Galactic plane (Liszt \& Spiker 1995; Yusef-Zadeh, Hewitt \& Cotton 2004). At the core of the HII region, an elongated infrared dark cloud is noted in which two maser sources and a green source are embedded. Figure 2a shows contours of submm emission at $850 \mu \mathrm{m}$ (Pierce-Price et al. 2000) superposed on an IRAC image of this region at $8 \mu \mathrm{m} .4 .5 \mu \mathrm{m}$ contours of the bright source which shows prominently in a three-color image of Sgr C are shown in Figure $2 \mathrm{~b}$. The crosses show the positions of methanol maser emission at $6.7 \mathrm{GHz}$. As pointed out earlier both these maser sources could be associated with the green source in Sgr C. We have also mapped 44-GHz methanol maser emission from the green source using Haystack Observatory. This transition of methanol maser emission, which is known to be collisionally pumped, is identified on a scale of $1^{\prime}$ oriented along the extent of the major axis of the green source. This is also consistent with a picture in which the green source is associated with the maser emission source in Sgr C (a more detailed account of this source will be given elsewhere).

We have modelled the SED of the green source G359.436-0.102 in Sgr C and have estimated the mass and luminosity of the central protostar coincident with the norther maser source by fitting the SED from a large grid of 2-D YSO models (Robitaille et al. 2006, 2007). The models include a disk and an accreting envelope with bipolar cavities. Figure 3a shows the best fitted models to the data using IRAC, MIPS and submm data accounting for 30 magnitudes of visual extinction. Due to the lower resolution of submm data, we show upper limits for the peak flux densities at 850 and $450 \mu \mathrm{m}$ (PiercePrice et al. 2000) at the position of the green source. Different curves of the model fits correspond to the variation of the observed apertures. These model fits all seem to be consistent with the SED of the source. Figure 3b shows the derived mass and luminosity of the green source based on the well-fit SED models. This fit of the SED is consistent with the identification of the green source with a massive protostar, associated with the methanol masers. The derived parameters of protostars using green sources with methanol maser counterparts should be useful indicators of the evolutionary phase of these sources. In addition, further study of the green/maser sources may also address whether linear distribution of maser spots come from circumstellar disks or outflows (De 

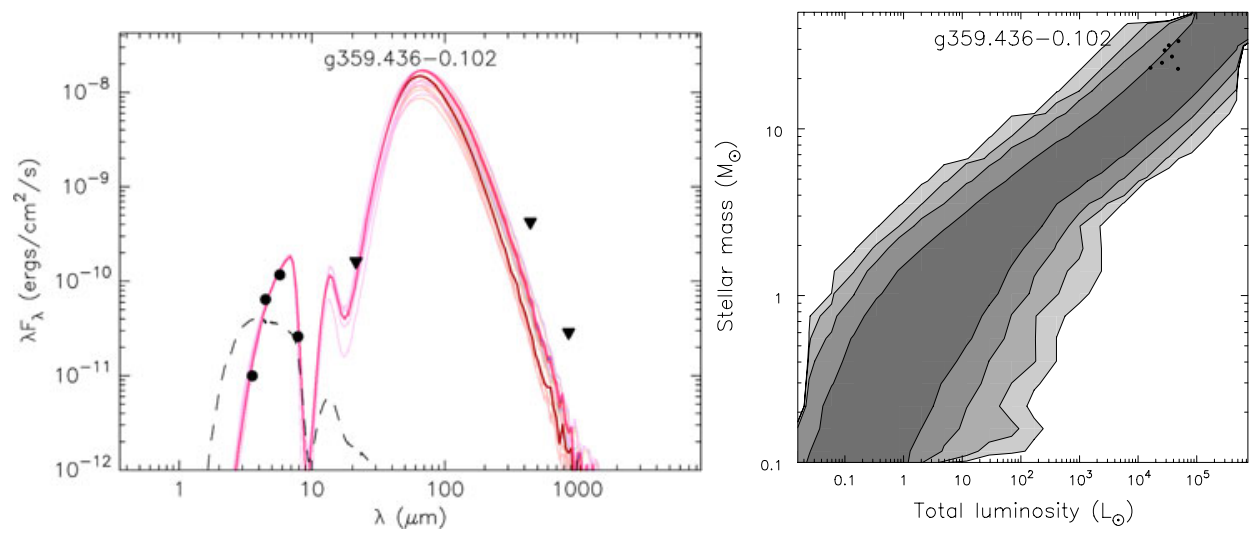

Figure 3. (a) A preliminary SED of the northern maser G359.436-0.102 in Sgr C and the fitted model with different aperture sizes. (b) The grey scale shows distribution of stellar mass and luminosity from a grid of 20,000 YSO radiative transfer models. The black dots show the mass and luminosities of the well-fit SEDs (those in Figure 3a).

Buizer et al. 2005). Furthermore, green sources found based on IRAC observations can provide an extensive target list for future methanol maser studies.

\section{The role of cosmic rays}

We believe that the low on-going star formation activity inferred from the estimate of the supernova rate and the rarity of methanol masers can be explained by enhanced cosmic ray flux in the nuclear disk. High cosmic-ray fluxes in molecular clouds affect star formation by heating the gas and increasing its ionization fraction (Yusef-Zadeh, Wardle \& Roy 2007a). Higher cloud temperatures increase the Jeans mass, potentially changing the IMF, while high ionization increases magnetic coupling to the cloud material, reducing ambipolar diffusion and increasing the time taken for gravitationally unstable cores to contract to the point that they overwhelm their magnetic support.

Several studies have recently indicated the importance of cosmic rays in the nuclear disk. One is from the detection of low frequency $74 \mathrm{MHz}$ radio emission from the central region of the Galaxy, indicating enhanced cosmic rays with cosmic ray electron density of $\sim 7 \mathrm{eV} \mathrm{cm}^{-3}$ (LaRosa et al. 2005). The other is strong $\mathrm{H}_{3}^{+}$absorption lines detected along several lines of sight towards the Galactic center (Oka et al. 2005). These absorption lines are much stronger than those seen in the Galactic disk. These measurements infer that the ionization rate in the inner 30pc of the Galaxy is more two orders of magnitude higher than that that in the Galactic disk. The high ionization rate is more likely to be due to high cosmic ray flux as revealed by high synchrotron emissivity in raido wavelengths than due to diffuse hot X-ray emitting gas. Lastly, the large scale distribution of K $\alpha$ Iron emission line at $6.4 \mathrm{keV}$ associated with a number of cloud complexes in the nuclear disk has been correlated with the distribution of nonthermal radio emission from this region (Yusef-Zadeh et al. 2007b). The energy density of the cosmic rays required to explain the $6.4 \mathrm{keV} \mathrm{X}$-ray emission from the inner $2^{0} \times 0.5^{0}$ of the molecular nuclear disk ranges between 20 and $10^{3} \mathrm{eV} \mathrm{cm}^{-3}$.

\section{Acknowledgements}

We thank Y. Pihlström for providing us with Figure 1a. C.O.H. is supported by the Lindheimer Postdoctoral Fellowship. 


\section{References}

Caswell, J. L. 1996, MNRAS 283, 606

Candon, J. J. 1992, ARA\&A 30, 575

Cox, P., \& Laureijs, R. 1989, The Center of the Galaxy, 136, 121

De Buizer, J. M., Radomski, J. T., Telesco, C. M., \& Piña, R. K. 2005, ApJ Supp, 156, 179

Frail, D. A. et al. 1996, AJ 111, 1651

Gaensler, B. M. et al. 2004, ApJ, 616, 383

Ghez, A. M., et al. 2003, ApJ, 586, L127

Gusten, R., \& Downes, D. 1981, A\&A, 99, 27

Hüttemeister, S. et al. 1993, A\& $A$ 280, 255

Karlsson et al. 2003, A\&SA 403, 1011

LaRosa, T. N. et al. 2005, $A p J$ 626, L23

Lis, D. C.\& Carlstrom, J. E. 1994, ApJ 424, 189

Lis, D. C.\& Menten, K. 1998, ApJ 507, 794

Liszt, H.S., \& Spiker, R.W. 1995, ApJS 98, 259

Lockett, P., Gauthier, E. \& Elitzur, M. 1999, ApJ 511, 235

Marston et al. 2004, ApJS 154, 333

Menten, K. M. 1991, ApJ, 380, L75

Morris, M. \& Serabyn, E. 1996, ARA\& $\&$ 34, 645

Mori, H. et al. 2004, 35th COSPAR Scientific Assembly, 35, 3705

Nord et al. 2004, $A J$ 128, 1646

Odenwald, S. F., \& Fazio, G. G. 1984, ApJ, 283, 601

Oka, T. et al. 2005, ApJ 632, 882

Paumard, T. et al. 2006, ApJ 643, 1011

Pedlar et al. 1989, ApJ 342, 769

Pierce-Price, D. et al. 2000, ApJ 545, L121

Pihlström, Y. M. \& Sjouwerman, L. O. 2006, JPhCS 54, 77

Reach, W. T. 2006, AJ 131, 1479

Robitaille, T. P., Whitney, B. A., Indebetouw, R., Wood, K., \& Denzmore, 2006, ApJS 167, 256

Robitaille, T. P., Whitney, B. A., Indebetouw, R. \& Wood, K. 2007, ApJS (in press, astro$\mathrm{ph} / 0612690)$

Schödel, R., Ott, T., Genzel, R., Eckart, A., Mouawad, N., \& Alexander, T. 2003, ApJ, 596, 1015

Smith, H., Hora, J. L., Marengo, M. \& Pipher, J. L. 2006, ApJ 645, 1264

Stolovy, S. et al. 2006, JPhCS 54, 176

Tsuboi, M., Okumura, S. K. \& Miyazaki, A. 2006, JPhCS 54, 16

Uchida et al. 1992, ApJ 398, 128

Wardle, M. 1999, ApJ 525, L101

Yusef-Zadeh, F., Roberts, D. A., Bower, G., Wardle, M., \& Goss, W. M. 2002, Cosmic Masers: From Proto-Stars to Black Holes, 206, 212

Yusef-Zadeh, F., Hewitt, J. W., \& Cotton, W. 2004, ApJS 155, 421

Yusef-Zadeh, F., Muno, M., Wardle, M. \& Lis, D. C. 2007b, ApJ 656, 847

Yusef-Zadeh, F., Uchida, K. I. \& Roberts, D. 1995, Science 270, 1801

Yusef-Zadeh, F., Roberts, D. A., Goss, W. M., Frail, D. A., \& Green, A. J. 1996, ApJ, 466, L25

Yusef-Zadeh, F.; Roberts, D. A.; Goss, W. M.; Frail, D. A.; Green, A. J. 1999, ApJ 512, 230

Yusef-Zadeh, F., Wardle, M. \& Roy, S. 2007a, ApJ submitted 